\title{
From Triazine to Heptazine: the Origin of Graphitic Carbon Nitride as a Photocatalyst
}

Nan Liu, ${ }^{\dagger}$ Tong Li,${ }^{\dagger}$ Ziqiong Zhao, ${ }^{\dagger}$ Jing Liu,,$^{\dagger}$ Xiaoguang Luo, ${ }^{\ddagger}$ Xiaohong Yuan, ${ }^{\S}$ Kun Luo, ${ }^{\dagger, \perp}$

Julong He, ${ }^{\dagger}$ Dongli $Y u,{ }^{* \dagger}$ and Yuanchun Zhao ${ }^{\dagger}{ }^{\dagger}$

${ }^{\dagger}$ State Key Laboratory of Metastable Materials Science and Technology, Yanshan University, Qinhuangdao 066004, China

${ }^{\ddagger}$ Department of Electronics, College of Electronic Information and Optical Engineering, Nankai University, Tianjin 300071, China

${ }^{\S}$ Center for High Pressure Science and Technology Advanced Research, Beijing 100094, China

${ }^{\perp}$ Hebei Key Laboratory of Microstructural Material Physics, School of Science, Yanshan University, Qinhuangdao 066004, China

E-mail: ydl@ysu.edu.cn (D.L.Y.); yzhao56@ysu.edu.cn (Y.C.Z.). 
Table S1. Reported Space Group, Atom Number $(Z)$ in the Unit Cell, Lattice Parameters, Unit Cell Volume ( $V$ ) of Melamine, Melam, and Melem

\begin{tabular}{lcccccccc}
\hline structure & space group & $Z$ & $a(\AA)$ & $b(\AA)$ & $c(\AA)$ & $\beta\left(^{\circ}\right)$ & $V\left(\AA^{3}\right)$ & ref. \\
\hline melamine & $P 2_{1} / \mathrm{c}($ no. 14) & 4 & 7.27 & 7.48 & 10.57 & 112.33 & 531.69 & $\mathrm{~S} 1$ \\
melam & $C 2 / c($ no. 15) & 12 & 18.11 & 10.87 & 13.98 & 96.31 & 2735.40 & $\mathrm{~S} 2$ \\
melem & $P 2_{1} / \mathrm{c}$ (no. 14) & 4 & 7.40 & 8.65 & 13.38 & 99.91 & 843.95 & $\mathrm{~S} 3$ \\
\hline
\end{tabular}

Table S2. Calculated Lattice Parameters, Unit Cell Volume $(V)$, Total Energy $\left(E_{\text {total }}\right)$, and Relative Error of the Volume $\left(R_{V}\right)$ with Respect to the Experimental Value of Melamine, Melam, and Melem

\begin{tabular}{lccccccc}
\hline structure & $a(\AA)$ & $b(\AA)$ & $c(\AA)$ & $\beta\left(^{\circ}\right)$ & $V\left(\AA^{3}\right)$ & $E_{\text {total }}(\mathrm{eV} /$ atom $)$ & $R_{V}(\%)$ \\
\hline melamine & 7.14 & 7.41 & 10.57 & 113.20 & 513.36 & -146.04 & -3.45 \\
melam & 17.92 & 10.80 & 14.24 & 96.10 & 2741.12 & -156.17 & +0.21 \\
melem & 7.65 & 8.39 & 13.53 & 101.78 & 850.48 & -169.99 & +0.77 \\
\hline
\end{tabular}

Table S3. Calculated Band Gaps of Melamine, Melam, and Melem Based on PBE-TS and the HSE06 Hybrid Functional, Respectively

\begin{tabular}{lcc}
\hline \multirow{2}{*}{ structure } & \multicolumn{2}{c}{ band gap $(\mathrm{eV})$} \\
\cline { 2 - 3 } & PBE-TS & HSE06 \\
\hline melamine & 4.21 & 5.53 \\
melam & 3.39 & $-{ }^{a}$ \\
melem & 3.29 & 4.38 \\
\hline
\end{tabular}

${ }^{a}$ HSE06 calculation for the melam structure has failed for several times due to its large unit cell (12 molecules and 312 atoms in total). 

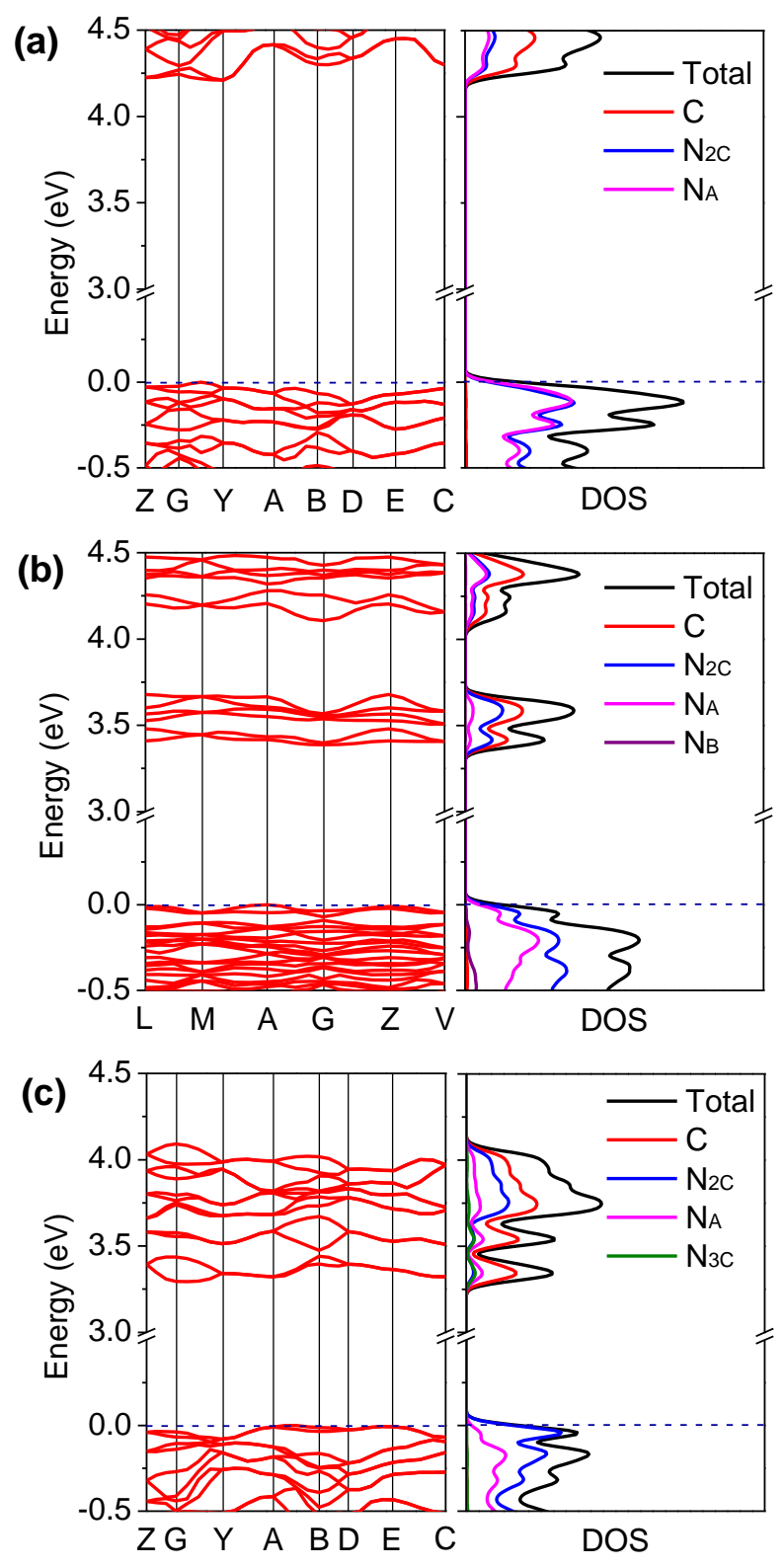

Figure S1. Details near the valence band maximum (VBM) and the conduction band minimum (CBM) of the calculated band structures and density of states of (a) melamine, (b) melam, and (c) melem, respectively. The results displayed here are enlarged from those shown in Figure 1. 


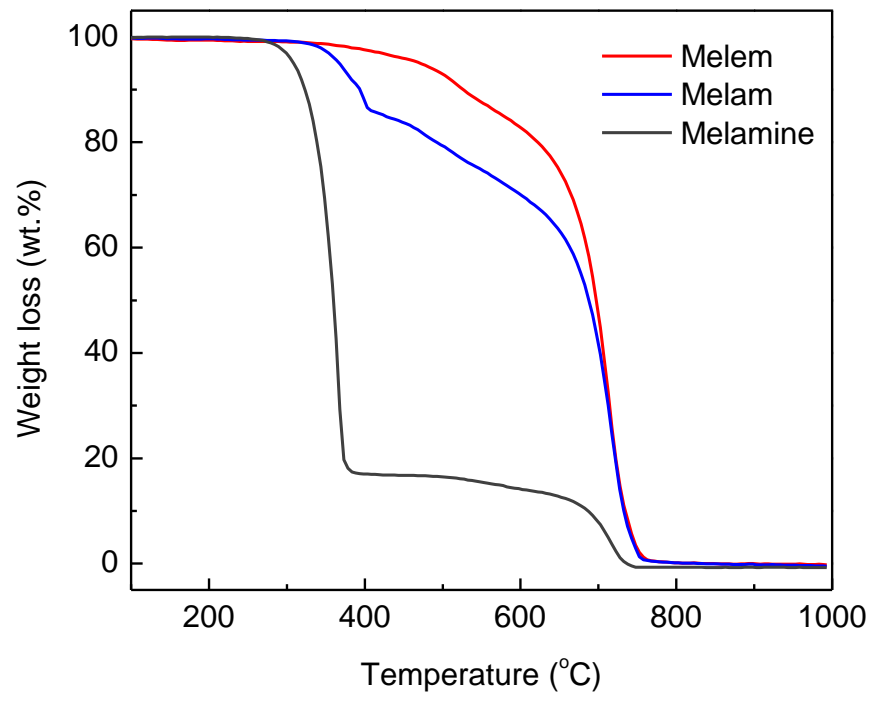

Figure S2. Thermogravimetric analysis (TGA) curves of melamine, melam, and melem.

Table S4. Raw Data of Elemental Analyses, the Calculated C/N and C/H Atomic Ratios of Melamine, Melam, and Melem, Respectively

\begin{tabular}{lcccccc}
\hline compound & measurements & $\mathrm{C}(\mathrm{wt} \%)$ & $\mathrm{N}(\mathrm{wt} \%)$ & $\mathrm{H}(\mathrm{wt} \%)$ & $\mathrm{C} / \mathrm{N}$ (atomic) & $\mathrm{C} / \mathrm{H}$ (atomic) \\
\hline \multirow{3}{*}{ melamine } & $\# 1$ & 28.56 & 66.54 & 4.792 & 0.5003 & 0.5002 \\
& $\# 2$ & 58.51 & 66.60 & 4.718 & 0.4990 & 0.5071 \\
& $\# 3$ & 28.59 & 66.76 & 4.624 & 0.4992 & 0.5189 \\
\hline \multirow{3}{*}{ melam } & $\# 1$ & 30.08 & 64.39 & 3.658 & 0.5446 & 0.6901 \\
& $\# 2$ & 31.10 & 64.62 & 3.352 & 0.5610 & 0.7786 \\
& $\# 3$ & 30.62 & 64.43 & 3.763 & 0.5540 & 0.6829 \\
\multirow{3}{*}{ melem } & $\# 1$ & 32.65 & 63.38 & 2.453 & 0.6005 & 1.1170 \\
& $\# 2$ & 32.59 & 63.14 & 2.597 & 0.6017 & 1.0532 \\
& $\# 3$ & 32.83 & 62.73 & 2.525 & 0.6101 & 1.0912 \\
\hline
\end{tabular}



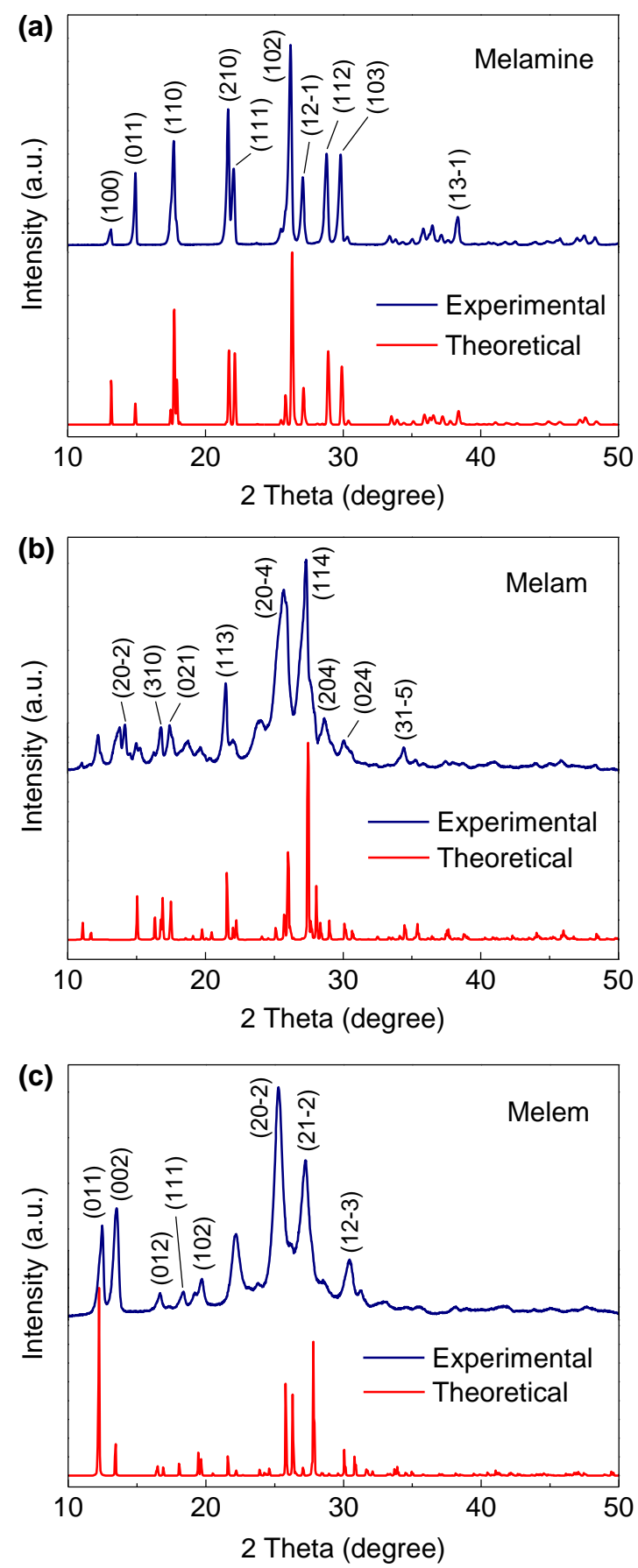

Figure S3. Comparison between the experimental and theoretical XRD patterns of (a) melamine, (b) melam, and (c) melem. The measured patterns have been indexed, and the theoretical patterns were generated by the Reflex package of Materials Studio, based on the proposed crystal structures as presented in Table S1. 
Table S5. Details of the Fitting Results for the High-Resolution C1s and N1s XPS Spectra of Melamine, Melam, and Melem

\begin{tabular}{|c|c|c|c|c|c|c|c|}
\hline compound & & peak & $\begin{array}{c}\text { position } \\
(\mathrm{eV})\end{array}$ & $\begin{array}{l}\text { height } \\
\text { (CPS) }\end{array}$ & $\begin{array}{c}\text { FWHM } \\
(\mathrm{eV})\end{array}$ & $\begin{array}{c}\text { area } \\
(\mathrm{CPS} . \mathrm{eV})\end{array}$ & area ratio \\
\hline \multirow{7}{*}{ melamine } & \multirow{3}{*}{$\mathrm{C} 1 \mathrm{~s}$} & $\mathrm{C}_{\mathrm{ad}}$ & 284.6 & 5562.40 & 1.40 & 8114.33 & 0.27 \\
\hline & & $\mathrm{C}_{3 \mathrm{~N}}$ & 287.5 & 24957.04 & 1.14 & 29604.97 & 1.00 \\
\hline & & $\pi$ excitation & 294.1 & - & - & - & - \\
\hline & \multirow{4}{*}{ N1s } & $\mathrm{N}_{2 \mathrm{C}}$ & 398.1 & 38631.18 & 1.28 & 51510.61 & 1.00 \\
\hline & & $\mathrm{N}_{\mathrm{A}}$ & 399.1 & 34987.30 & 1.34 & 48685.62 & 0.95 \\
\hline & & $\mathrm{N}_{\mathrm{A} 2}$ & 399.7 & 3738.11 & 1.55 & 6052.27 & 0.12 \\
\hline & & $\pi$ excitation & 405.0 & - & - & - & - \\
\hline \multirow{8}{*}{ melam } & \multirow{3}{*}{$\mathrm{C} 1 \mathrm{~s}$} & $\mathrm{C}_{\mathrm{ad}}$ & 284.6 & 6740.15 & 1.45 & 10190.17 & 0.25 \\
\hline & & $\mathrm{C}_{3 \mathrm{~N}}$ & 287.6 & 28593.33 & 1.36 & 40568.88 & 1.00 \\
\hline & & $\pi$ excitation & 293.7 & - & - & - & - \\
\hline & \multirow{5}{*}{ N1s } & $\mathrm{N}_{2 \mathrm{C}}$ & 398.1 & 45327.94 & 1.27 & 59986.18 & 1.00 \\
\hline & & $\mathrm{N}_{\mathrm{B}}$ & 398.7 & 11288.38 & 0.95 & 11144.64 & 0.19 \\
\hline & & $\mathrm{N}_{\mathrm{A}}$ & 399.4 & 22656.36 & 1.56 & 36877.60 & 0.61 \\
\hline & & $\mathrm{N}_{\mathrm{A} 2}$ & 400.6 & 2718.07 & 0.99 & 2801.05 & 0.05 \\
\hline & & $\pi$ excitation & 404.4 & - & - & - & - \\
\hline \multirow{8}{*}{ melem } & \multirow{3}{*}{$\mathrm{C} 1 \mathrm{~s}$} & $\mathrm{C}_{\mathrm{ad}}$ & 284.6 & 5676.09 & 1.42 & 8387.35 & 0.18 \\
\hline & & $\mathrm{C}_{3 \mathrm{~N}}$ & 287.9 & 37442.80 & 1.20 & 46817.88 & 1.00 \\
\hline & & $\pi$ excitation & 293.5 & - & - & - & - \\
\hline & \multirow{5}{*}{ N1s } & $\mathrm{N}_{2 \mathrm{C}}$ & 398.3 & 55089.17 & 1.22 & 69920.85 & 1.00 \\
\hline & & $\mathrm{N}_{3 \mathrm{C}}$ & 398.9 & 10355.20 & 1.01 & 10867.75 & 0.16 \\
\hline & & $\mathrm{N}_{\mathrm{A}}$ & 399.5 & 17248.19 & 1.78 & 32076.70 & 0.46 \\
\hline & & $\mathrm{N}_{\mathrm{A} 2}$ & 400.9 & 6713.23 & 1.07 & 7490.98 & 0.11 \\
\hline & & $\pi$ excitation & 404.2 & - & - & - & - \\
\hline
\end{tabular}



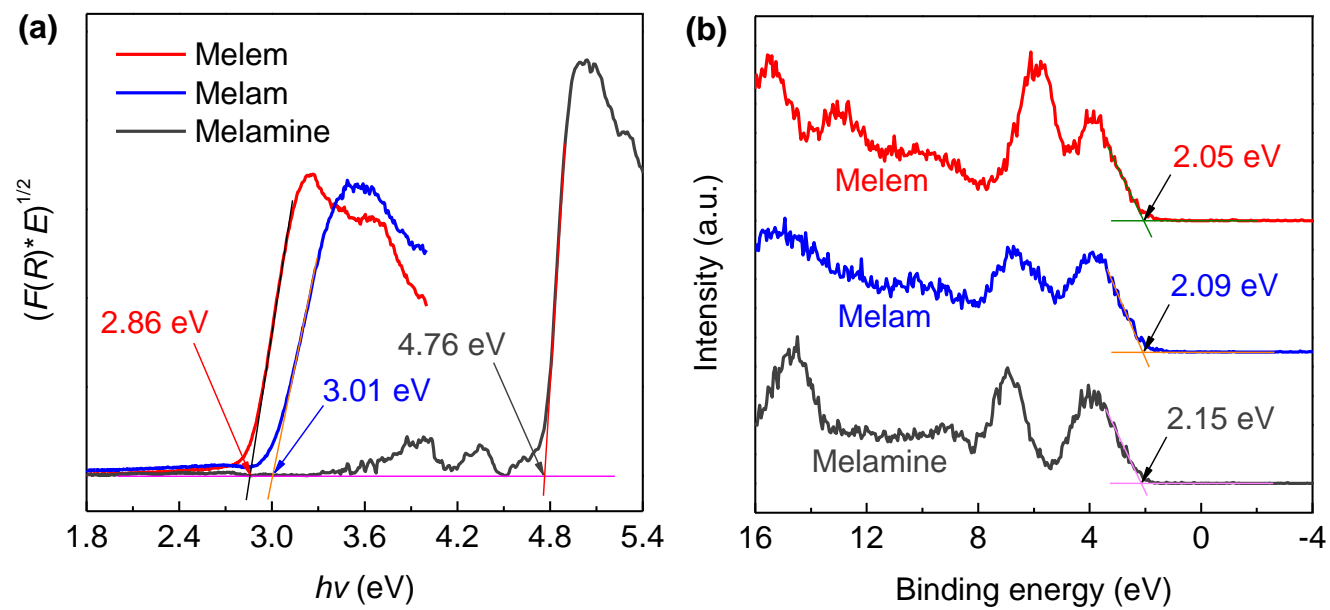

Figure S4. (a) Kubelka-Munk plots the measured UV-vis diffuse reflectance spectra and (b) XPS valence band spectra of melamine, melam, and melem, respectively.

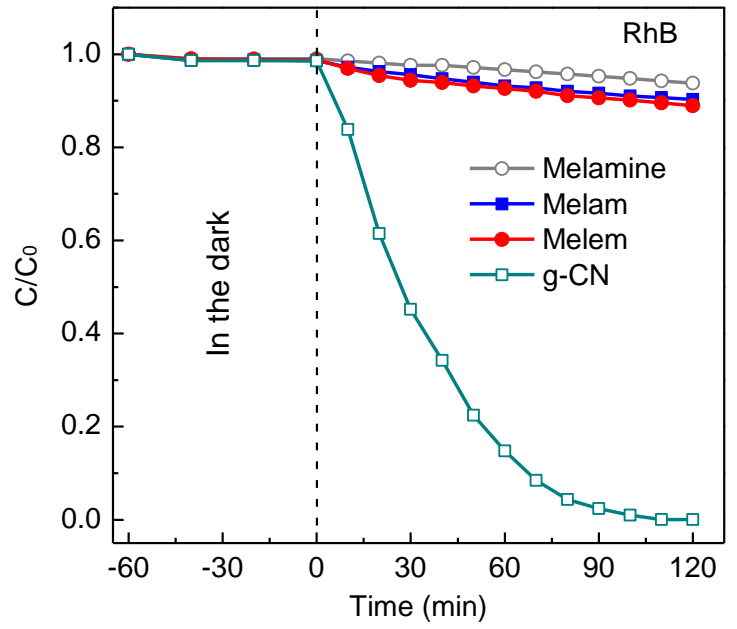

Figure S5. Photocatalytic activities for RhB degradation of melamine, melam, melem, and polymeric g-CN under visible light irradiation $(\lambda>420 \mathrm{~nm})$. Polymeric $\mathrm{g}-\mathrm{CN}$ was synthesized by thermal condensation of melamine at $550{ }^{\circ} \mathrm{C}$ for $4 \mathrm{~h}$ under a flowing nitrogen atmosphere, the experimental details and characterization can be found in our previous work. ${ }^{\mathrm{S}}$ 

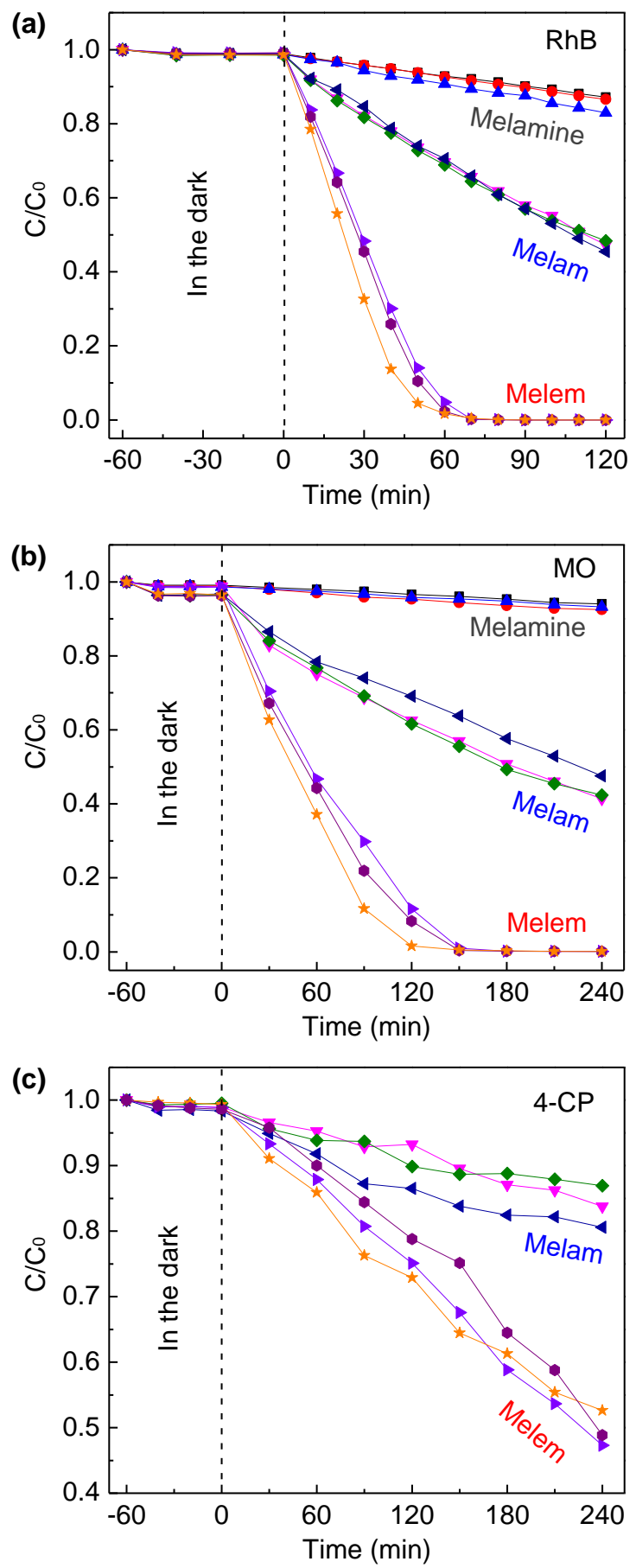

Figure S6. Raw data of the photodegradation of (a) RhB, (b) MO, and (c) 4-CP catalyzed by different samples. The degradation processes were conducted under UV-visible light irradiation $(\lambda>300 \mathrm{~nm})$. 

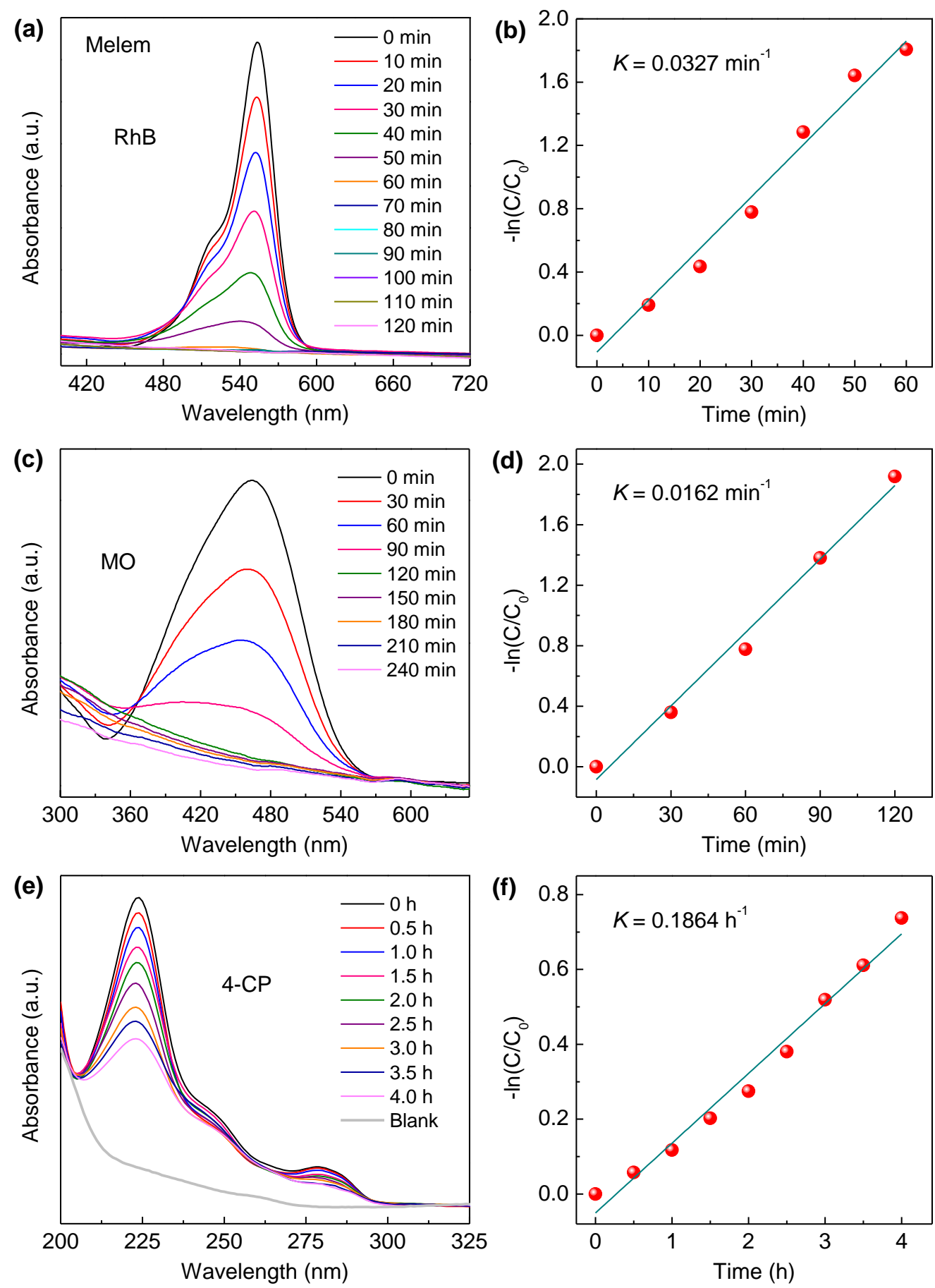

Figure S7. Typical time-dependent absorption spectra showing the degradation process and the corresponding first-order degradation constant $(K)$ for $(\mathrm{a}, \mathrm{b}) \mathrm{RhB}$, (c,d) $\mathrm{MO}$, and $(\mathrm{e}, \mathrm{f})$ 4-CP catalyzed by melem under UV-visible light irradiation $(\lambda>300 \mathrm{~nm})$. The blank spectrum in (e) was recorded from pure deionized water as a reference. 


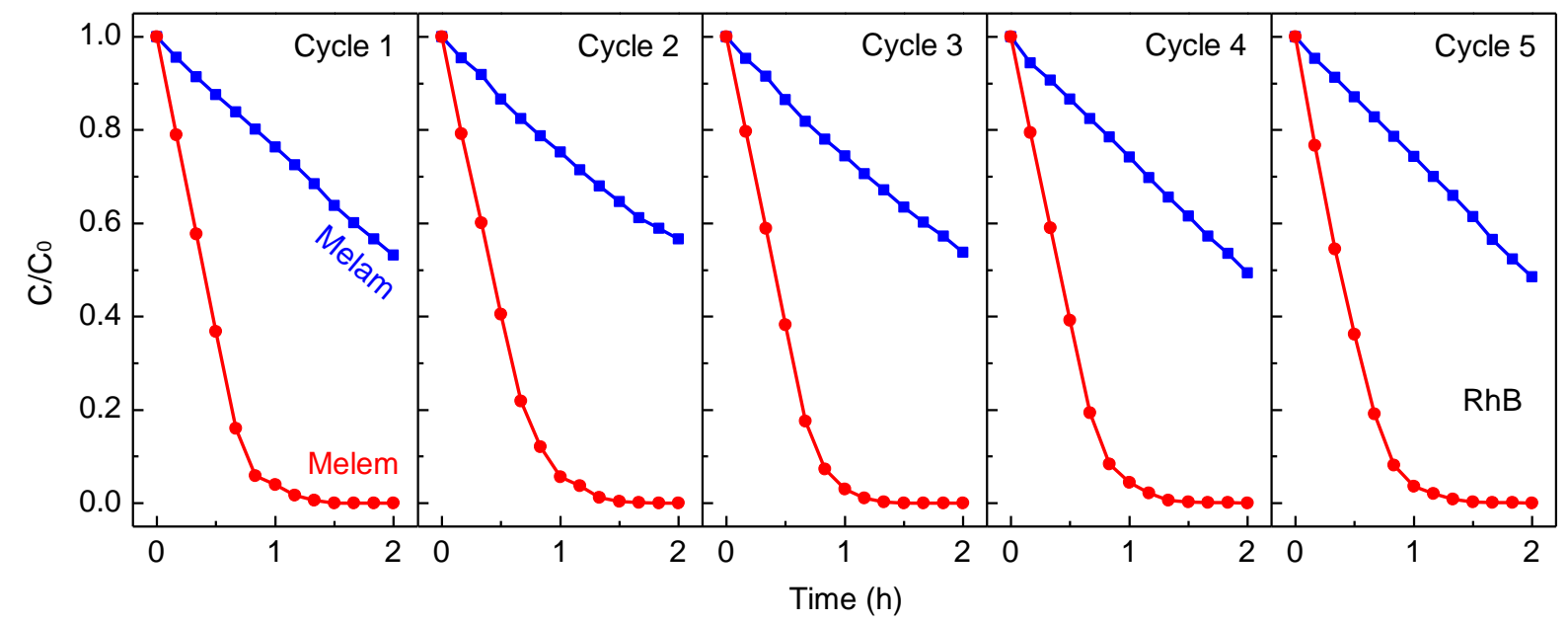

Figure S8. Photocatalytic stability of melam and melem evaluated by recycling degradation of RhB under UV-visible light irradiation $(\lambda>300 \mathrm{~nm})$. 

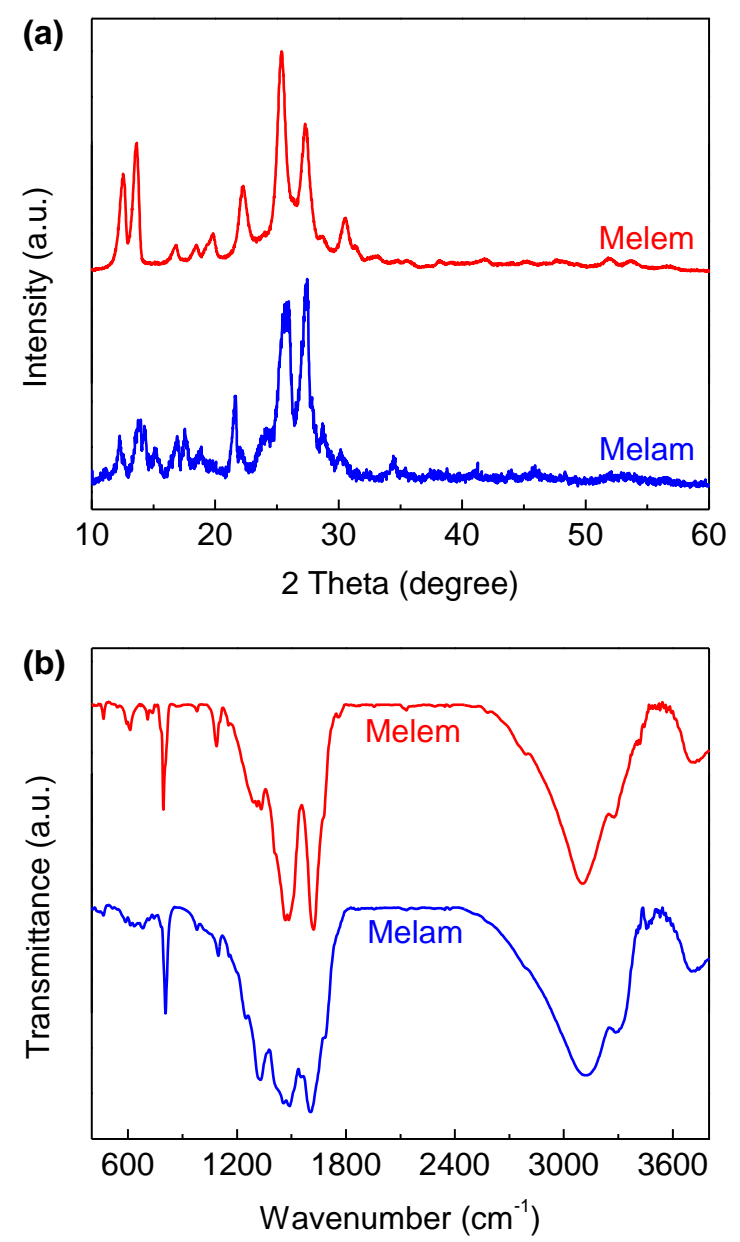

Figure S9. (a) XRD patterns and (b) FTIR spectra of melam and melem after the recycled degradation tests of $\mathrm{RhB}$. The catalyst powders were re-collected by centrifugation, washed carefully by deionized water for several times, and dried in an oven at $200{ }^{\circ} \mathrm{C}$ overnight. 


\section{References}

(S1) Yuan, X.; Luo, K.; Zhang, K.; He, J.; Zhao, Y.; Yu, D. Combinatorial Vibration-Mode Assignment for the FTIR Spectrum of Crystalline Melamine: A Strategic Approach toward Theoretical IR Vibrational Calculations of Triazine-Based Compounds. J. Phys. Chem. A 2016, $120,7427-7433$.

(S2) Lotsch, B. V.; Schnick, W. New Light on an Old Story: Formation of Melam during Thermal Condensation of Melamine. Chem. - Eur. J. 2007, 13, 4956-4968.

(S3) Jürgens, B.; Irran, E.; Senker, J.; Kroll, P.; Müller, H.; Schnick, W. Melem (2,5,8Triamino-tri-s-triazine), an Important Intermediate during Condensation of Melamine Rings to Graphitic Carbon Nitride: Synthesis, Structure Determination by X-ray Powder Diffractometry, Solid-State NMR, and Theoretical Studies. J. Am. Chem. Soc. 2003, 125, 10288-10300.

(S4) Wu, J.; Ji, X.; Yuan, X.; Zhao, Z.; Li, Y.; Wen, B.; Zhang, H.; Yu, D.; Zhao, Y.; Tian, Y. Regulating Polymerization in Graphitic Carbon Nitride To Improve Photocatalytic Activity. Chem. Mater. 2019, 31, 9188-9199. 